\title{
Standard Model Treatment of the Radiative Corrections to Neutron $\beta$-Decay
}

\section{G. G. Bunatian}

Joint Institute for Nuclear

Research, 141980, Dubna, Russia
Starting with the Standard Model electroweak Lagrangian, the radiative corrections to neutron $\beta$-decay are obtained. Nucleon compositeness is considered by appropriate parameterization of the nucleon weak transition current and electromagnetic form factors.
Key words: neutron $\beta$-decay; radiative corrections.

Accepted: August 11, 2004

\section{Introduction and Discussion}

The present treatment [1] of the neutron $\beta$-decay,

$$
\mathrm{n} \Rightarrow \mathrm{p}+\mathrm{e}^{-}+\bar{v}+\gamma,
$$

is based on the Standard Model electroweak Lagrangian [2-5]

$$
\mathcal{L}^{\mathrm{EW}}\left(A_{\mu}, Z_{\mu}, W_{\mu}^{ \pm}, H, \psi_{\mathrm{f}}, e, M_{\mathrm{Z}}, M_{\mathrm{W}}, M_{\mathrm{H}}, m_{\mathrm{f}}, \xi\right),
$$

which specifies the propagators of electromagnetic, $Z-, W^{ \pm}$-boson, Higgs, and fermion fields, and the interactions between these fields. The quantities $e=\sqrt{4 \pi \alpha}, M_{\mathrm{Z}}, M_{\mathrm{W}}, M_{\mathrm{H}}, m_{\mathrm{f}}$ are the unit of charge, masses of the Z-boson, W-boson, Higgs-boson, and fermions, respectively; the Feynman gauge $\xi=1$ is chosen. In calculating the neutron $\beta$-decay amplitude in the one-loop approach, we leave out the effects of Higgs-fermion interactions, since they are of the order of the Higgs coupling to fermions $\approx m_{\mathrm{f}} / M_{\mathrm{W}}[2-5]$. Only the first generations of leptons $\left(\mathrm{e}, v_{\mathrm{e}}\right)$ and quarks ( $u$-, $d$-quarks) come into the consideration.

The transition amplitude $\mathcal{M}$ of the process in Eq. (1), when calculated in the one-loop approach directly in terms of the bare fields and parameters, is UV-divergent, and renormalization is necessary. The multiplicative renormalization of the Lagrangian in Eq. (2) is performed amenably to the non-minimal on-mass-shell (OMS) renormalization scheme [3-5]. Upon calculating the radiative corrections with the fields, masses, and coupling constants renormalized within the OMS renormalization scheme, the UV divergencies occurring in the loop expansion (of propagators as well as $S$-matrix elements) are absorbed in the infinite parts of the renormalization constants. Also the finite parts of the radiative corrections are fixed. These lead to physically observable consequences. 
As the nucleon is a composite system of strong interacting quarks, the amplitude $\mathcal{M}$ of the process in Eq. (1) is determined by

$$
\begin{gathered}
\mathcal{M} \cdot i(2 \pi)^{4} \delta\left(P_{\mathrm{n}}-P_{\mathrm{p}}-p_{\mathrm{e}}-p_{\mathrm{v}}-p_{\gamma}\right)= \\
\left\langle\Phi_{0 \mathrm{p}}^{\mathrm{q}+}\left(P_{\mathrm{p}}, \sigma_{\mathrm{p}}\right), \phi_{\mathrm{e}}^{+}\left(p_{\mathrm{e}}, \sigma-e\right), A\left(p_{\gamma}\right)\left|\boldsymbol{S}_{\mathrm{int}}\right| \Phi_{0 \mathrm{n}}^{\mathrm{q}}\left(P_{\mathrm{n}}, \sigma_{\mathrm{n}}\right), \phi_{\mathrm{v}}\left(-p_{\mathrm{v}},-\sigma_{\mathrm{e}}\right)\right\rangle, \\
\text { where } \quad \boldsymbol{S}_{\mathrm{int}} \equiv \boldsymbol{S}_{\mathrm{int}}(\infty,-\infty)=\mathcal{T} \exp \left(i \int \mathrm{d}^{4} x \mathcal{L}_{\text {int }}(x)\right)
\end{gathered}
$$

is dictated by the general Lagrangian $\mathcal{L}_{\text {int }}(x)=\mathcal{L}_{\text {int }}^{\mathrm{EW}}(x)+\mathcal{L}_{\text {str }}^{q q}(x)$, incorporating both electroweak and strong interactions. Here $\Phi_{0 \mathrm{p}, 0 \mathrm{n}}^{q}, \phi_{\mathrm{e}, v}$, and $A$ stand to describe the quark systems, electrons, neutrinos and $\gamma$ rays. Nowadays, there seems no option but to allow for the effect of strong interactions by introducing the baryon weak and electromagnetic form factors. The Born amplitude $\mathcal{M}^{0}$, represented by the first diagram in Eq. (6), is written in terms of the bare vertexes $\Gamma^{\mathrm{evW}}, \Gamma^{\mathrm{npW}}(k)$ and bare W-propagator $D^{\mathrm{W}}(k)$, depicted by the point, blob, and thin wavy line. As the momentum transfer $k^{2} \ll M_{N}^{2}$, we actually deal with

$$
\Gamma_{\alpha}^{\mathrm{npW}}(0)=\frac{e\left|V_{\mathrm{ud}}\right|}{2 \sqrt{2 s_{\mathrm{W}}}} \gamma_{\alpha}\left(1-g_{\mathrm{A}}(0) \gamma^{5}\right), D_{\alpha \beta}^{\mathrm{W}}(0)=\frac{-g_{\alpha \beta}}{M_{\mathrm{W}}^{2}}, s_{\mathrm{W}}^{2}=1-\frac{M_{\mathrm{W}}^{2}}{M_{\mathrm{Z}}^{2}},
$$

and the electromagnetic form factors $f_{\alpha}^{p p}(0)=\gamma_{\alpha}, f_{\alpha}^{n n}(0)=0$. The corrected renormalized amplitude $\mathcal{M}$ is presented in the one-loop approach by the set of diagrams.
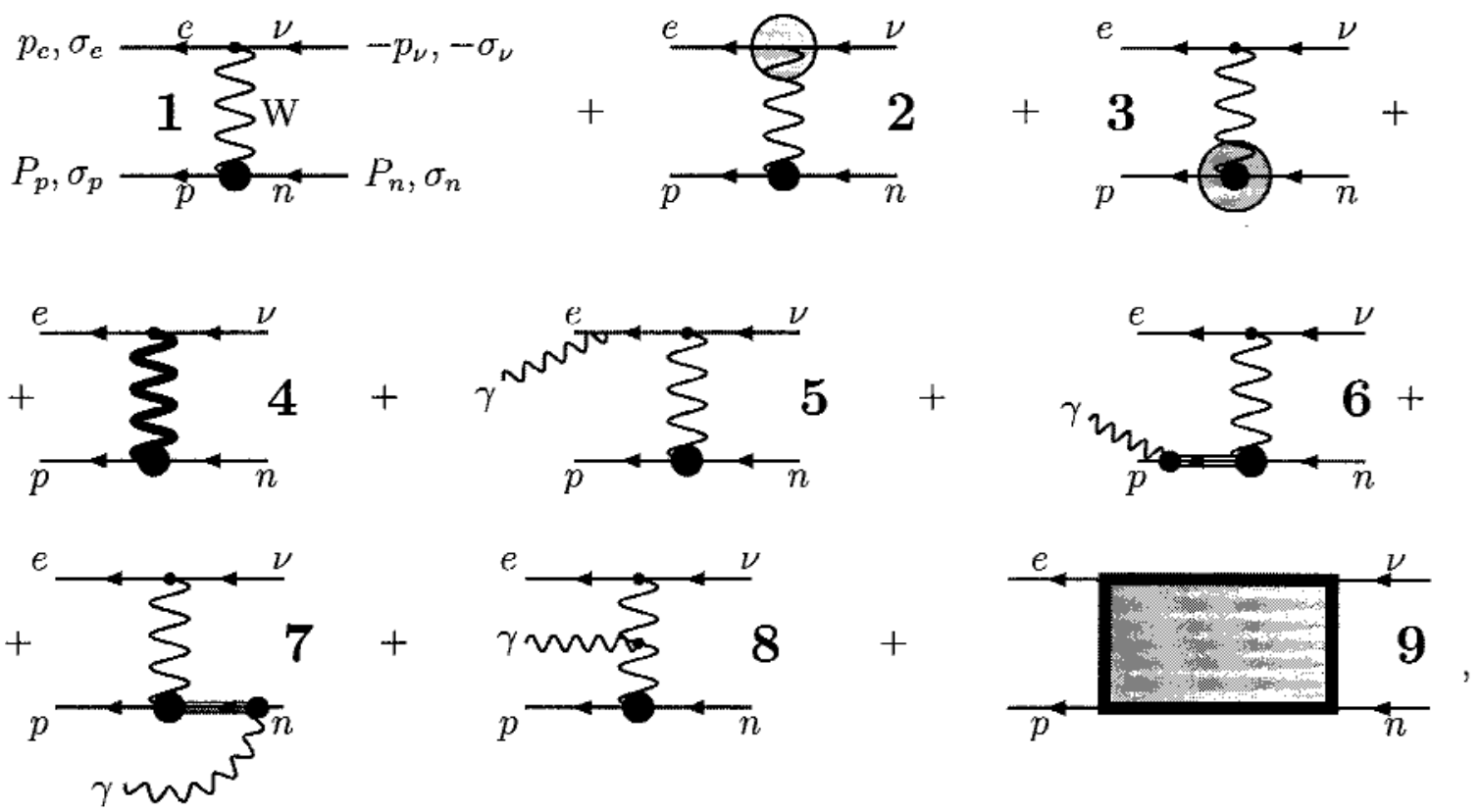

Calculation of the one-loop leptonic vertex $\hat{\Gamma}^{e v \mathrm{w}}$, depicted by the shaded circle in the diagram 2, is straightforward and results in merely a multiplicative factor to $\Gamma^{e v \mathrm{~W}}$. Next, from $\mu$-decay analysis [4], we obtain the corrected renormalized $\mathrm{W}$-propagator $\hat{D}^{\mathrm{W}}$. depicted by the heavy wavy line in the diagram 4 , which replaces $D^{\mathrm{W}}$ in the diagram 1. As $m, M_{\mathrm{n}}-M_{\mathrm{p}} \ll M_{\mathrm{p}} \ll M_{\mathrm{w}}$, the contributions from the diagrams 6 through 8 are negligible as compared to one coming out of the diagram 5, which renders the common bremsstrahlung of a final electron [6]. 
To treat strong interactions which are inherent in the processes described by the corrected renormalized vertex (the $\hat{\Gamma}^{\mathrm{npw}}(k)$ shaded circle with heavy core in the diagram 3 ), and by the irreducible four-fermion amplitude $\mathcal{M}_{2 \gamma}$ (the "box-diagrams" 9), we split the virtual photon propagator, involved therein, into two parts

$$
\begin{gathered}
D_{\mu \nu}^{A \lambda}\left(x_{2}-x_{3}\right)=g_{\mu v} \int \frac{\mathrm{d}^{4} k}{(2 \pi)^{4}}\left(\frac{1}{k^{2}-M_{\mathrm{S}}^{2}+i 0}+\frac{-M_{\mathrm{S}}^{2}}{\left(k^{2}-\lambda^{2}+i 0\right)\left(k^{2}-M_{\mathrm{S}}^{2}+i 0\right)}\right) \mathrm{e}^{-i k\left(x_{2}-x_{3}\right)}= \\
=D_{\mu \nu}^{A s}\left(x_{2}-x_{3}\right)+D_{\mu \nu}^{A l}\left(x_{2}-x_{3}\right)
\end{gathered}
$$

where the subsidiary matching parameter $M_{\mathrm{S}}, M_{\mathrm{p}}^{2} \ll M_{\mathrm{S}}^{2} \ll M_{\mathrm{W}}^{2}[2,7]$, emerges to separate large, $k^{2} \geqslant M_{\mathrm{S}}^{2}$, and comparatively small, $k^{2}<M_{\mathrm{S}}^{2}$, momenta transferred by the virtual photon. Then, the quantities $\hat{\Gamma}^{n \tilde{N}^{\prime}}, \mathcal{M}_{2 \gamma}$ are divided into two parts incorporating these "massive" and "soft" photons with the propagators $D^{A s}, D^{4 l}$, respectively,

$$
\hat{\Gamma}_{\alpha}^{n p W}=\hat{\Gamma}_{s \alpha}^{n p W}+\hat{\Gamma}_{l \alpha}^{n p W}, \quad \mathcal{M}_{2 \gamma}=\mathcal{M}_{2 \gamma s}+\mathcal{M}_{2 \gamma l} .
$$

In the quantities $\hat{\Gamma}_{\mathrm{s}}^{n p W}, \mathcal{M}_{2 \gamma}$, the electroweak interactions mediated by Z- and W-bosons and "massive" photons transfer the large momenta $k^{2} \gtrsim M_{\mathrm{S}}^{2}$ to a quark system, so that strong quark-quark interactions die out, and quarks become asymptotically free in the respective intermediate states. Consequently, calculation of $\hat{\Gamma}^{n p W}, M_{2}$ descends to evaluation of the transition matrix elements between the neutron $\left|\Phi_{\mathrm{n}}^{q}\left(P_{\mathrm{n}}, \sigma_{\mathrm{n}}\right)\right\rangle$ and proton $\left\langle\Phi_{\mathrm{p}}^{q}\left(P_{\mathrm{p}}, \sigma_{\mathrm{p}}{ }^{\gamma s}\right)\right.$ states of the expressions given in terms of free quark operators:

$$
\begin{aligned}
& \left(\bar{u}_{u}\left(p_{u}, \sigma_{u}\right) \hat{\Gamma}_{\mathrm{S} \alpha}^{u d W}\left(p_{u}, p_{d}, q\right) w^{+\alpha}(q) u_{d}\left(p_{d}, \sigma_{d}\right)\right)= \\
& =p_{u}, \sigma_{u} \stackrel{W^{+} \sum^{q} d}{u} p_{d}, \sigma_{d}+
\end{aligned}
$$
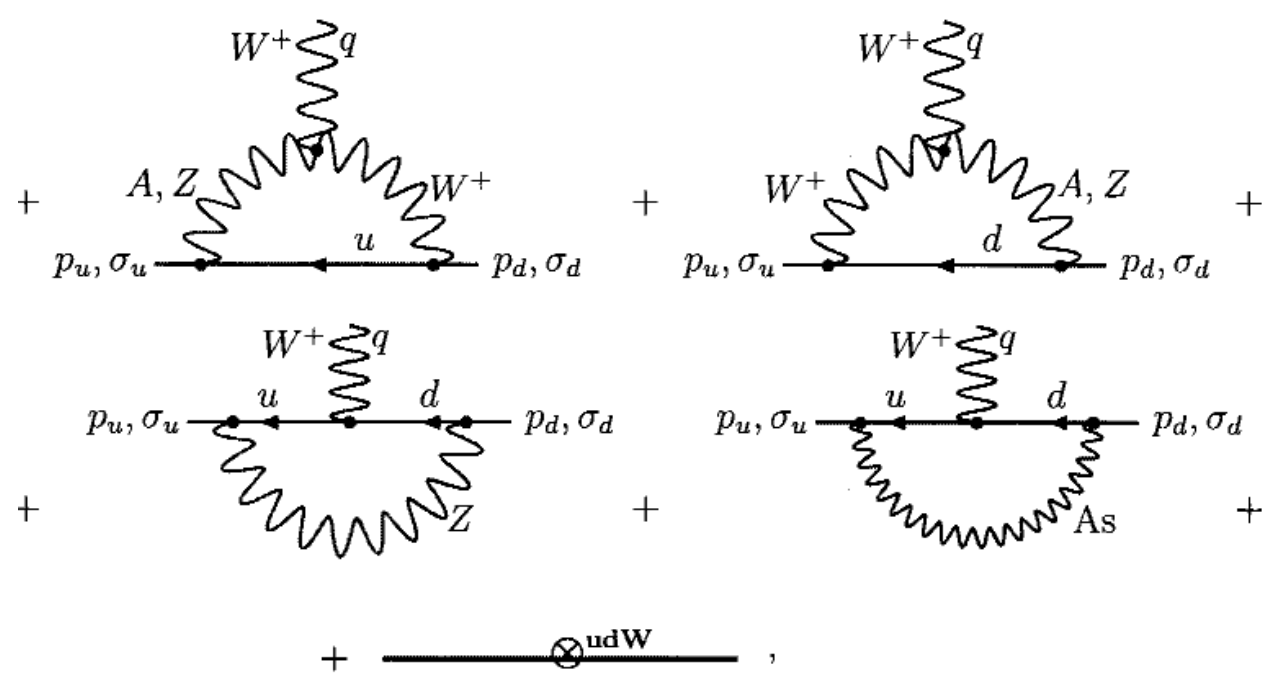
in the case of $\hat{\Gamma}_{\mathrm{s}}^{n p W}$, and $\left(\bar{\psi}_{\mathrm{e}}(x) \bar{\psi}_{u}(x) \hat{\Gamma}^{e v u d} \psi_{d}(x) \psi_{v}(x)\right)=$

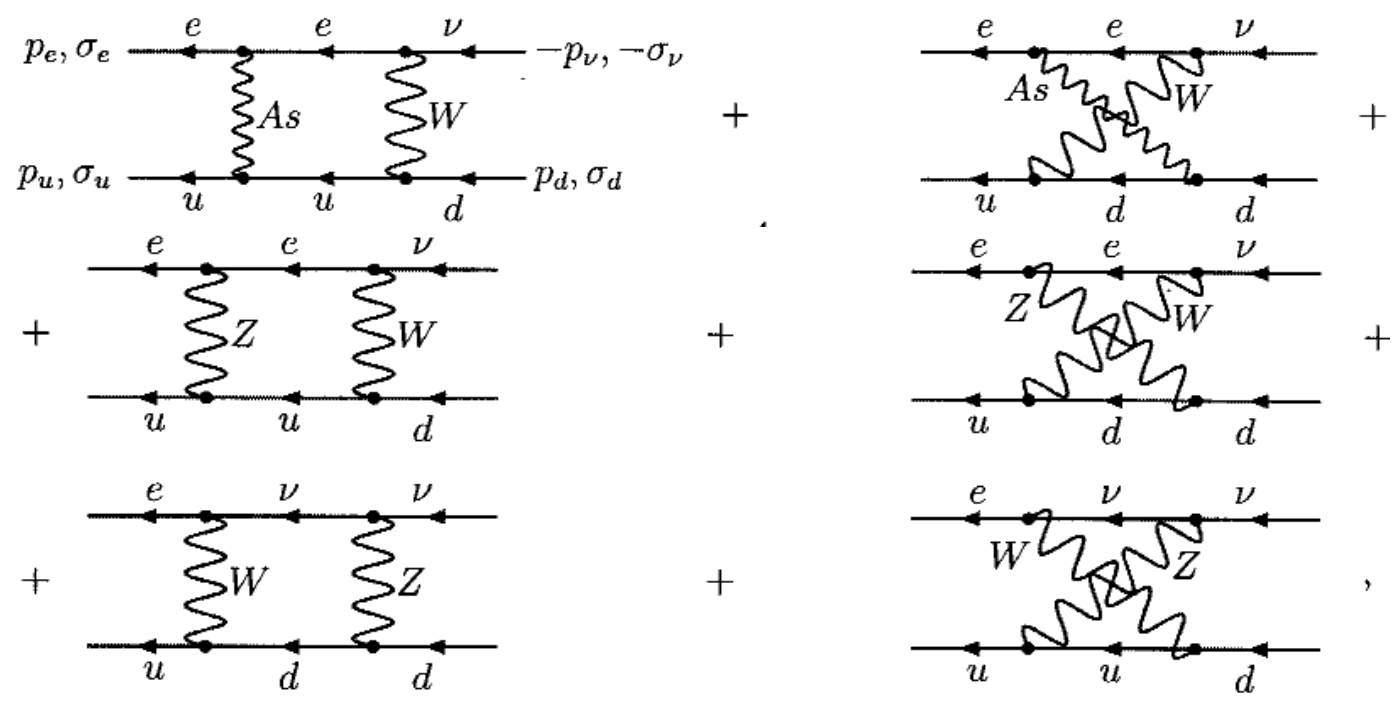

in evaluating $\mathcal{M}_{2 \gamma s}$. The wavy line with the tag $A s$ stands for the "massive photon" propagator $D_{A s}$ from Eq. (7) Eventually, $\hat{\Gamma}_{\mathrm{s}}^{n p W^{2}}, \mathcal{M}_{2 \gamma s}$ prove merely to be proportional to $\Gamma^{n p W}, \mathcal{M}^{0}$.

In the vertex $\hat{\Gamma}_{l}^{n p W}$ and in the amplitude $\mathcal{M}_{2 \gamma l}$, the "soft" photons transfer comparatively small momenta to a quark system, so that quarks constitute the baryon in such intermediate states. Actually, the prevailing parts of $\hat{\Gamma}_{l}^{n p W}, \mathcal{M}_{2 \gamma l}$ are obtained by retaining only pure single nucleon intermediate states and presuming the vertexes and form factors found in Eq. (5). Then, $\hat{\Gamma}_{l \alpha}^{p n W} \approx \delta z^{p} \cdot \Gamma_{\alpha}^{n p W}(0) / 2$, where the UV-finite renormalization constant $\delta z^{p}$ of the proton wave function is defined in terms of the proton self-energy caused by "soft" photons of Eq. (7). The amplitude $\mathcal{M}_{2 \gamma l}$ results in

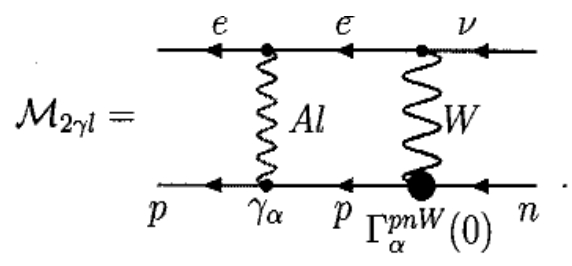

The wavy line with the tag $A l$ stands for the "soft" photon propagator $D^{4 l}$ in Eq. (7). The quantity $\mathcal{M}_{2 \gamma l}$ of Eq. (11) turns out to be not a multiple of the Born amplitude $\mathcal{M}^{0}$.

For now, strong interactions remain an unsatisfied calculational challenge. To realize the precision of the calculations, we size up how they are affected by allowance for (I) the contribution from the diagrams
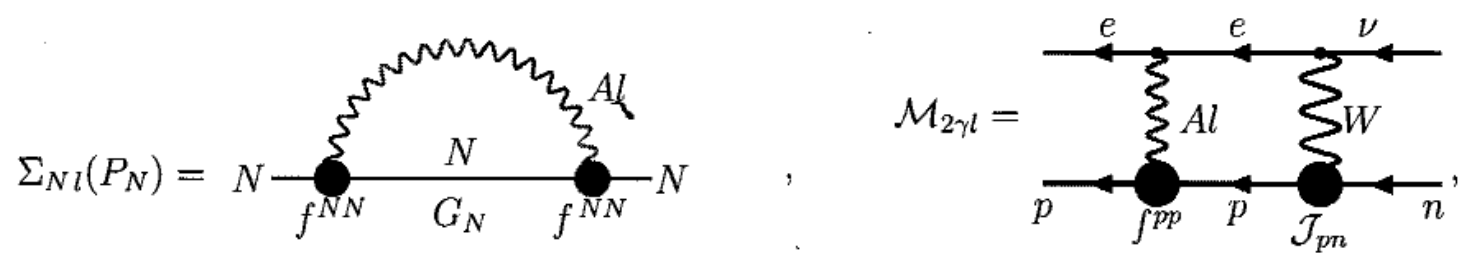
with the nucleon common transition currents and form factors

$$
\begin{gathered}
J_{\mathrm{np}}^{\beta}(k)=\gamma^{\beta} g_{\mathrm{V}}\left(k^{2}\right)+g_{\mathrm{WM}}\left(k^{2}\right) \sigma^{\beta v} k_{\mathrm{v}}-\left(\gamma^{\beta} g_{\mathrm{A}}\left(k^{2}\right)+g_{\mathrm{PP}}\left(k^{2}\right) k^{\beta}\right) \gamma^{5}, \\
f_{\alpha}^{N N}(k)=f_{1}^{N N}\left(k^{2}\right) \gamma_{\alpha}+f_{2}^{N N}\left(k^{2}\right) k^{\beta} \sigma_{\alpha \beta}, \quad g_{\mathrm{WM}} \approx \frac{\mu_{\mathrm{n}}-\mu_{\mathrm{p}}}{2 M_{\mathrm{p}}} \approx-\frac{3.7}{2 M_{\mathrm{p}}}, g_{\mathbb{P}}\left(k^{2}\right) \sim \frac{8 g_{\mathrm{A}}(0)}{2 M_{\mathrm{p}}}, \\
f_{1}^{p p}\left(k^{2}\right) \approx \frac{-m_{\mathrm{p}}^{2}}{k^{2}-m_{\mathrm{p}}^{2}}, f_{2}^{p p}\left(k^{2}\right) \approx \frac{1.79}{2 M_{\mathrm{p}}} \frac{-m_{\mathrm{p}}^{2}}{k^{2}-m_{\mathrm{p}}^{2}}, f_{1}^{n n}=0, \quad f_{2}^{n n}\left(k^{2}\right)=\frac{1.93}{2 M_{\mathrm{p}}} \frac{m_{\mathrm{p}}^{2}}{k^{2}-m_{\mathrm{p}}^{2}},
\end{gathered}
$$

and also (II) by allowance for insertion of the $\Delta_{33}$-isobar instead of the nucleon in the intermediate states in the diagrams
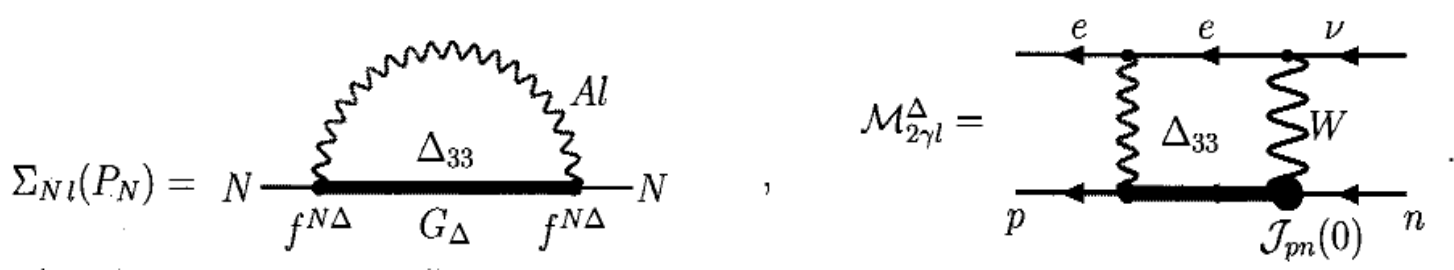

The thereby entailed effects prove to amount to about a few tenths of a percent, $\approx(0.1-0.3) \%$, to the value of decay amplitude $\mathcal{M}$. These estimations present the ambiguities inherent in the calculations. In fact, that is what restricts, together with the uncertainties $\approx 0.1 \%$ due to the parameter $M_{\mathrm{S}}$ entanglement, the accuracy attainable in the present treatment of the neutron $\beta$-decay, without additional physical fit parameters, besides $g_{\mathrm{A}},\left|V_{\text {ud }}\right|$, involved.

With the amplitude $\mathcal{M}$ from Eq.(6) as described above, the electron momentum distribution

$$
\mathrm{d} \boldsymbol{W}\left(\varepsilon, \boldsymbol{p}_{\mathrm{e}}, \boldsymbol{\xi}\right)=\mathrm{d} \boldsymbol{w}\left(\left(\varepsilon, \boldsymbol{p}_{\mathrm{e}}, \xi\right), \boldsymbol{p}_{\mathrm{e}}\right)\left\{W_{0}\left(g_{\mathrm{A}}, \varepsilon\right)+v \xi W_{\xi}\left(g_{\mathrm{A}}, \varepsilon\right)\right\}
$$

turns out not to be a multiple of the quantity

$$
\begin{gathered}
\mathrm{d} \boldsymbol{W}^{0}\left(\varepsilon, \boldsymbol{p}_{\mathrm{e}}, \boldsymbol{\xi}\right)=\mathrm{d} \boldsymbol{w}\left(\varepsilon, \boldsymbol{p}_{\mathrm{e}}\right)\left(1+3 g_{\mathrm{A}}^{2}+v \xi 2 g_{\mathrm{A}}\left(1-g_{\mathrm{A}}\right)\right), \\
\mathrm{d} \boldsymbol{w}\left(\varepsilon, \boldsymbol{p}_{\mathrm{e}}\right)=\frac{G^{2}\left|V_{\mathrm{ud}}\right|^{2}}{2 \pi^{3}} \varepsilon\left|\boldsymbol{p}_{\mathrm{e}}\right| k_{\mathrm{m}}^{2} \mathrm{~d} \varepsilon \frac{\mathrm{d} \boldsymbol{n}}{4 \pi}, \boldsymbol{n}=\boldsymbol{p}_{\mathrm{e}} /|\boldsymbol{p}|, \boldsymbol{v}=\boldsymbol{p}_{\mathrm{e}} / \varepsilon, k_{\mathrm{m}}=M_{\mathrm{n}}-M_{\mathrm{p}}-\varepsilon,
\end{gathered}
$$

evaluated with the Born amplitude $\mathcal{M}^{0}$, unlike what was asserted in the investigations of Refs. [8-10]. Let us note that Eq. (12) comprises all the $\alpha$-order radiative corrections, without discarding the Coulomb term and separating the so called "model independent" and "model dependent" parts. Also, we nowhere appeal to the investigations of the $0^{+} \rightarrow 0^{+}$superallowed transitions in nuclei. If anything, introducing the new functions $\lambda^{\prime}\left(\varepsilon, \boldsymbol{p}_{\mathrm{e}}, g_{\mathrm{A}}\right), \lambda^{\prime \prime}\left(\varepsilon, \boldsymbol{p}_{\mathrm{e}}, g_{\mathrm{A}}\right)$, one might rewrite $W_{0}=1+3 \lambda^{\prime 2}, W_{\xi}=2 \lambda^{\prime \prime}\left(1-\lambda^{\prime \prime}\right)$. Yet as $\lambda^{\prime} \neq \lambda^{\prime \prime}$, it would be of no avail at all. The uncorrected asymmetry factor of the electron momentum distribution $A_{0}$ is replaced by the quantity $A(\varepsilon)$ accounting for the radiative corrections,

$$
A_{0}=\frac{2 g_{\mathrm{A}}\left(1-g_{\mathrm{A}}\right)}{1+3 g_{\mathrm{A}}^{2}} \Rightarrow \frac{W_{\xi}\left(g_{\mathrm{A}}, \varepsilon\right)}{W_{0}\left(g_{\mathrm{A}}, \varepsilon\right)}=A\left(\varepsilon, g_{\mathrm{A}}\right), \frac{A\left(\varepsilon, g_{\mathrm{A}}\right)-A_{0}}{A_{0}}=\delta A(\varepsilon) \approx-0.02( \pm \leqslant 0.002) \text {. }
$$


With $g_{\mathrm{A}}$ obtained, the radiative corrections cause the relative modification of the total decay probability $W$

$$
\frac{\int_{\mathrm{m}}^{M_{\mathrm{n}}-M_{\mathrm{p}}} \mathrm{d} \varepsilon \varepsilon\left|\boldsymbol{p}_{\mathrm{e}}\right| k_{\mathrm{m}}^{2} W_{0}\left(g_{\mathrm{A}}, \varepsilon\right)}{\left(1+3 g_{\mathrm{A}}^{2}\right) \int_{\mathrm{m}}^{M_{\mathrm{n}}-M_{\mathrm{p}}} \mathrm{d} \varepsilon \varepsilon\left|\boldsymbol{p}_{\mathrm{e}}\right| k_{\mathrm{m}}^{2}}-1=\delta W \approx 0.086( \pm \lesssim 0.003) .
$$

With the parameters obtained from $[4,5,11]$, the CKM matrix element

$$
\left|V_{\text {ud }}\right|^{2}=\frac{5335}{\tau_{\exp }\left(1+3 g_{\mathrm{A}}^{2}\right)(1+\delta W)},
$$

where $g_{\mathrm{A}}$ is determined by the $A_{\exp }$ value accordingly Eq. (14) and we directly arrive at

$$
2 g_{\mathrm{A}}\left(1-g_{\mathrm{A}}\right)=A_{\exp }(1-\delta A)\left(1+3 g_{\mathrm{A}}^{2}\right) .
$$

With the average values $\tau_{\text {exp }}=885.7 \mathrm{~s}, A_{\text {exp }}=-0.1162$ from Ref. [11], we find $g_{\mathrm{A}}=1.2729$ and $\left|V_{\text {ud }}\right|^{2}=0.9464$. With $A_{\text {exp }}=-0.1189$ ascertained in Ref. [12], the evaluation gives $g_{\mathrm{A}}=1.2804$ and $\left|V_{\text {ud }}\right|^{2}=0.9372$. It is to remark that presuming the value $\tau_{\text {exp }}=878 \mathrm{~s}$, reported in Ref. [13], we gain $\left|V_{\text {ud }}\right|^{2}=0.9545$ with the average value $\tau_{\text {exp }}=885.7 \mathrm{~s}$; with the values $\tau_{\text {exp }}=878 \mathrm{~s}$ and $A_{\exp }=-0.1189$ from Refs. $[12,13]$, we arrive at $\left|V_{\text {ud }}\right|^{2}=0.9453$. As observed thereof, the deficiencies $\Delta \approx-0.003-0.013$ could be expected in the relation $\left|V_{\mathrm{ud}}\right|^{2}+\left.\left|V_{\mathrm{us}}^{2}+\right| V_{\mathrm{ub}}\right|^{2}=1-\Delta$, with the average values $\mid V_{\text {us }}{ }^{2} \approx 0.0482$ and $\left|V_{\text {ub }}\right|^{2} \approx 2 \cdot 10^{-5}$ from Ref. [11]. Considering these evaluations, we are to behold that their precision is about a few tenth of percent, $\leq 0.5 \%$, as expounded above. So, strictly speaking, there seems no profound reason to assert an evidence of the CKM-unitarity violation, with accounting for the errors inherent in the $\tau_{\exp }$ and $A_{\text {exp }}$ values themselves as well [11-13].

Having at our disposal the amplitude $\mathcal{M}$ of Eq. (6), we obtain [14] the modification of the recoil proton momentum distribution caused by the radiative corrections

$$
\begin{gathered}
\delta\left(\left|P_{\mathrm{p}}\right|, g_{\mathrm{A}}\right)=\delta\left(\frac{\mathrm{d} W_{\mathrm{p}}\left(\left|P_{\mathrm{p}}\right|, g_{\mathrm{A}}\right)}{\mathrm{d}\left|P_{\mathrm{p}}\right|} \frac{1}{W_{\mathrm{p}}\left(\left|P_{\mathrm{p}}\right|, g_{\mathrm{A}}\right)}\right) \approx(0.01-0.02), \\
\frac{\mathrm{d} W_{\exp }\left(\left|P_{\mathrm{p}}\right|\right)}{\mathrm{d}\left|P_{\mathrm{p}}\right|} \cdot \frac{1}{W_{\exp }\left(\left|P_{\mathrm{p}}\right|\right)}=\frac{W_{0}\left(\left|P_{\mathrm{p}}\right|, g_{\mathrm{A}}\right)}{\mathrm{d}\left|P_{\mathrm{p}}\right|} \cdot \frac{1}{W_{\mathrm{p}}\left(\left|P_{\mathrm{p}}\right|, g_{\mathrm{A}}\right)}\left(1+\delta\left(\left|P_{\mathrm{p}}\right|, g_{\mathrm{A}}\right)\right),
\end{gathered}
$$

which offers an additional condition to ascertain the $g_{\mathrm{A}}$ value from experimental data processing.

It should be noted that the final state of neutron $\beta$-decay given in Eq. (1) involves not three, but four particles because of the $\gamma$ rays. Much needed high precision measurements of electron and proton momentum distributions will provide additional information on the quantities $\left|V_{\text {ud }}\right|$ and $g_{\mathrm{A}}$, yet not the neutrino correlation coefficients $B, a$, and $D$ themselves [14-15].

Ingenious introduction of the redundant physical fit parameters, besides $g_{\mathrm{A}},\left|V_{\text {ud }}\right|$, to describe the effects of nucleon compositeness is believed to provide advancement in study the neutron $\beta$-decay.

\section{References}

[1] G. G. Bunatian, Radiative Corrections to the Neutron $\beta$-decay within the Standard Model, arXiv:hep-ph/0311350 (November 2003) [Accessed November 2003].

[2] J. F. Donoghue, E. Golowich, and B. R. Holstein, Dynamics of the Standard Model, Cambridge University Press, Cambridge, UK (1994).

[3] K. I. Aoki et al., Suppl. Progr. Theor. Phys. 73, 1 (1982).

[4] W. Hollik, Fortschr. Phys. 38, 165 (1990). 
[5] D. Bardin, G. Passarino, The Standard Model in the Making, Oxford, 1999

[6] G. G. Bunatian, Phys. Atomic. Nuc. 63, 502 (2000);/aps1999mar11_005.

[7] R. Decker and Finkemeier, Nucl. Phys. B 438, 17 (1996).

[8] A. Sirlin, Phys. Rev. 164, 1767 (1967).

[9] I. S. Towner and J. C. Hardy, J. Phys. G. 29, 197 (2003); Phys. Rev. C 66, 035501 (2002); Nucl. Phys. A 540 (1992).

[10] A. Sirlin, Radiative Corrections and the Universality of the Weak Interactions, arXiv:hep-ph/0309187.

[11] D. E. Groom et al., (PDG), Eur. Phys. J. C 15, 1 (2000).

[12] H. Abele et al., Phys. Rev. Lett. 88, 211801-1 (2002).

[13] V. Varlamov et al., Neutron Lifetime Experiment with Gravitational Trap and with Lower Temperature Fomblin (LTF) Coating, in this Special Issue.

[14] G. G. Bunatian, E4-2000-19 Preprint of JINR, Dubna, Russia (2000); /aps2000feb17_001. G. G. Bunatian, Part. and Nucl., Lett. No. 6[103]2000, 63 (2000).

[15] G. G. Bunatian, JETP 89, 811 (1999).

About the author: Gevorg G. Bunatian is a senior staff member in the Neutron Physics Laboratory of the Joint Institute for Nuclear Research, JINR, Dubna, Moscow region, Russia, 141980. 